\title{
Presentation of Pancreatic Cancer in a Dedicated Hepato- Pancreato-Biliary Surgery Unit of a Tertiary Health Facility, South-South, Nigeria: A Four Year Prospective Study.
}

\author{
OENgim $^{1}$, ADMarwa $^{2}$, MEkong $^{3}$, RNdoma-Egba $^{4}$ \\ ${ }^{1-4}$ Hepato-Pancreato-Biliary (HPB) Surgery Unit, Department of Surgery, University of Calabarand University \\ of CalabarTeaching Hospital, Calabar, Cross River State, Nigeria.
}

\begin{abstract}
Carcinoma of the pancreas is a common gastrointestinal neoplasm all over the world. It is the fourth most common cause of cancer related deaths in the United States of America. Though the disease is not as common in Africa compared to America and Europe, the incidence is however on the increase in Africa. Generally, the prognosis is poor especially in Africa, mainly because of late presentation.

We reviewed the pattern of presentation and management outcome of patients with obstructive jaundice $2^{0}$ to carcinoma of the pancreas from a prospectively kept data basein a dedicated Hepato-Pancreato-Biliary (HPB) Surgery Unit over a four year period (July, 2008 - June, 2012).A total of fourteen patients were seen with this condition during the study period. There were more females than males with a ratio of 2.5:1. The age range was 40-80years with a mean age of 58yrs. The commonest mode of presentation was a combination of jaundice, pruritus and weight loss. The presence of jaundice with pruritus were the principal symptoms that brought these patients to the hospital. Late presentation is common in our environment and prognosis is poor.
\end{abstract}

Keywords: Calabar, HPB surgery unit, obstructive jaundice, Pancreatic cancer

\section{Introduction}

Today, pancreatic adenocarcinoma is the fourth leading cause of cancer-related death in the U.S. and the western world ${ }^{1,2}$. Approximately $80 \%$ of patients present with unresectable disease due to the presence of metastases or local extension ${ }^{1}$. Pancreatic Cancer is also said to be the second most common gastrointestinal cancer and the fourth commonest cancer in males after lung cancer, prostate cancer and colorectal cancer in the western countries ${ }^{3}$. Though it is not as common in Africa compared to the western world, it can however not be said to be uncommon in our environment ${ }^{4}$.

This is the first prospective study on pancreatic cancer from this centre and is a reflection of the pattern of the disease in our environment. This paper aims to bring to the fore, the burden of this disease condition in this environment and add to the body of knowledge in the literature. We also aim to raise awareness on the nature of this condition as it affects our patients, most of whompresent late with attendant poor prognosis.

The incidence of this condition worldwide, almost equals its mortality even in the best of centers ${ }^{5}$. It is common after the age of forty but has been reported in children. The prognosis is generally poor and palliative treatment is often the rule as late presentation often makes curative resection (Whipples procedure) impossible ${ }^{1-6}$. At the time of diagnosis, $52 \%$ of all patients have distant metastasis and $26 \%$ of them already have locoregional spread $^{1}$. The relative one year survival of patients with this disease irrespective of stage is only $24 \%$ and the overall 5 year survival is $5 \%$. The median survival time for all patients with this disease is $4-6$ months irrespective of the stage ${ }^{1}$.

Pancreatic cancer can arise from either the exocrine portion of the gland (95\%) or from the endocrine portion $(5 \%)$. About $75 \%$ of these cancers occur within the head or neck of the gland,

$15-20 \%$ in the body and $5-10 \%$ in the tail of the gland ${ }^{1,2,5}$. These tumors can also arise from metastatic tumors from other sites such as the breast. The clinical presentation is largely influenced by the site of the tumour and the stage of the disease. Tumours of the head of pancreas are often associated with obstructive jaundice and pruritus at an early stage. Other symptoms irrespective of the site or stage of the tumour will include epigastric pain, anorexia, weight loss and abdominal distention from metastasis to the liver or ascites. Occasionally metastasis to the lungs will produce respiratory symptoms ${ }^{1-5}$. The risk factors include the black race, male gender, age above 40yrs, smoking, high intake of food rich in fat and proteins with little carbohydrate as well as exposure to certain pesticides and gasoline related chemicals ${ }^{1-5}$. Also new onset type II diabetes mellitus has been reported as a risk factor in $10-20 \%$ of cases. Investigative modalities necessary for diagnosis include urinalysis and liver function test. Abdominal ultrasonography scan, computed tomography scan of the abdomen, endoscopic retrograde cholangiopancreatography, diagnostic laparoscopy and endoluminal ultrasound scan with biopsy, all help to make a diagnosis in appropriate cases $^{1-5}$. Curative resection using the Whipple's procedure is only suitable for a few cases that may be diagnosed early. The majority of cases however can only 
have palliative surgical intervention such as the triple by-pass(cholecysto-jejunostomy, gastrojejunostomy and jejuno-jejunostomy) or a double by-pass (cholecysto-jejunostomy and gastro-jejunostomy). These by-pass procedures can also be done laparoscopically where appropriate expertise and facilities exist. Also palliative stenting of the biliary tree at ERCP can be done to relieve jaundice in advanced cases as well as use of duodenal stents to palliate gastric outlet obstruction. A percutaneous transhepatic cholangiography can also be used as a palliative modality. Adjuvant chemotherapy using gemcitabine or erlotinib have given marginal 1year survival of $24 \%^{3}$

\section{Methodology:}

A four year review (July, 2008 - June, 2012) of a prospectively kept data base of patients who were diagnosed clinically of obstructive jaundice $2^{0}$ to carcinoma of the pancreas was done. The starting date of this data corresponded to the establishment of this dedicated HPB Surgery Unit in this tertiary health facility. Their dermographic data, symptoms at presentation and examination findings were recorded. Relevant investigations were also done. Radiological evaluation was with either abdominal ultrasound scan, barium meal and follow through or abdominal CT-scan depending on what the patient could afford.

All the patients had abdominal ultrasound scan while only 6 patients (43\%) could afford a computerized tomography scan. Two patients $(14.3 \%)$ had a barium meal and follow through.

\section{Results:}

A total of fourteen patients were seen with this condition during the study period. There were more females than males with a ratio of 2.5:1. The age range was 40-80years with a mean age of 58yrs and a modal age of 60. The commonest mode of presentation was jaundice, pruritus and weight loss. The presence of jaundice with pruritus were the principal symptoms that brought these patients to the hospital. A positive family history was present in one female patient. Upper abdominal pain that often radiated to the back was present in seven patients (50\% of cases). The average duration of symptoms prior to presentation was 9 weeks, with a range of 6 weeks to 24weeks. . Advanced disease as evidenced by liver involvement, ascites or peritoneal deposits etc was seen in ten patients (71.4\%) while four patients (28.6\%) had no gross evidence of metastasis radiologically and at surgery.

All but one (93\%) of these patients had palliative surgical procedure while one female patient died of advanced disease before any surgical intervention. The commonest surgical procedure done was a triple by pass in ten patients (71.4\%) followed by a double by-pass in two patients (14.3\%) and a cholecyst-jejunostomy in one patient $(7.1 \%)$ because he was too ill to undergo double or triple by-pass. Pruritus was the earliest symptom to resolve in all the patients treated surgically. Amongst the 13 patients who had surgery, $8(62 \%)$ were discharged in stable condition from the hospital while 5 (38.5\%) died while on admission after surgery. All those who had surgery and were discharged from the hospital had resolution of the jaundice in the course of follow-up at the outpatient clinic except one male patient who continued to have these symptoms.

Nine $(69 \%)$ out of thirteen patients that had surgical intervention died within 18 months of surgery and five $(56 \%)$ of these patients died in the immediate post operative period. Only two (22\%) female patients who had no evidence of metastasis died between $12-18$ months after surgery while those who had metastasis especially to the liver died earlier. Four (31\%) of the patients who had surgery were lost to follow up.

\section{Discussion:}

The Literature on pancreatic Cancer from Nigeria is presently scanty compared with data from other parts of the world ${ }^{1,3}$. Wiredu et al ${ }^{7}$ found pancreatic cancer to be the fifth most common cause of cancer death in males following a ten year review of autopsies at the korle - Bu hospital in Accra.

Ajaoet $\mathrm{al}^{4}$ in Ibadan, Nigeria reported 36 proven cases of pancreatic cancer over a 4 year period (1975 - 1979) thus giving an average of 9 cases per year. The age range in their study was $17-78$ years with the highest incidence being above the age of 40 . They had a male preponderance ${ }^{4}$.Ajao et al cited a study from Rhodesia which reviewed 32 black African patients with this condition. The study, recorded a male preponderance with the disease being commoner between the ages of 40 and 60years in Rhodesia ${ }^{4}$. Onyekewere $\mathrm{et}^{3} \mathrm{a}^{3}$ in Lagos, Nigeria, reviewed all histologically proven pancreatic cancer seen over a two year period (20052007) in two hospitals in Lagos . A total of 27 cases were reported during their study period,also with a male preponderance. The age range in their study was 40-86 years with a mean age of 64 years $^{3}$. Alatise et al ${ }^{9}$ in IleIfe, Nigeria retrospectively reviewed the records of patients diagnosed of pancreatic cancer between 19892007 ,an 18year period. They had 96 cases though 80 were histologically confirmed. This gives an average of $4-$ 5 cases per year. There were more males than females in their series with a ratio of 2:1. The median age of the patients in their study was 55 years.

Whereas the studies cited above reported a higher male incidence, our present study however clearly shows a higher female preponderance $2.5: 1$ which is similar to the study by Erickson et $\mathrm{al}^{1}$ in the USA which 
reported a female preponderance. The reason for this higher female preponderance noticed in our environment is presently not clear. The higher number of patients recorded by Ajao and Onyekwere could be explained by the higher population in Ibadan and Lagos compared to Calabar which is less densely populated. Also, the study by Onyekwere et al was done using patients from two centres and this influenced the number of cases that were studied compared to the one centre study we did. However,all the Nigerian studies from different centres cited above ( Ajao et $\mathrm{al}^{4}$,Onyekwere et $\mathrm{al}^{3}$ and Alatise et $\mathrm{al}^{9}$ ) agree with the low incidence of this condition in Nigeria compared to the western world . Our present study also agrees with this low incidence in our environment.

The age incidence in our present study agrees largely with documentation in the literature where the disease is more common between 40-60years with a modal age of 60.In our study,the commonest mode of presentation was jaundice and pruritus. This agrees with documentation in the literature ${ }^{1-9}$. Onyekwereet $\mathrm{al}^{3}$ further stated that the pruritus is usually very distressing, thus necessitating their presentation in the hospital. This was also our experience in this series.

In our study, the average duration of symptoms before presentation was 9 weeks and this agrees with the late presentation observed by other workers around the world with its attendant poor prognosis ${ }^{1-9}$. The use of baseline investigations like urinalysis and liver function test in this condition cannot be over-emphasized as these when combined with clinical evaluation and abdominal ultrasound scan were most useful in making a diagnosis of this condition in our environment where diagnostic facilities are quite limited and expensive. At the earlier part of this study, abdominal CT Scan could only be done several kilometers away from our city at great cost. Only those who could afford it were able to do it (43\% of the patients) hence, abdominal ultrasound scan, barium meal and follow through were combined with clinical evaluation and relevant baseline investigations to arrive at a diagnosis. This scenario is always a reality in developing countries. Onyekwere et $\mathrm{al}^{3}$ were able to use laparoscopy and the tumour marker CA 19-9 in their study but these were unavailable in our centre during the study period. The use of ERCP, PTC, Endoscopic ultrasound scan, Laparoscopic surgery etc in the management of these patients are still far- fetched at present in most centres in Nigeria including our centre.

The high mortality rate recorded in our present study is in keeping with documentation in the literature $^{1-12}$. Apart from the 4 patients $(31 \%)$, who were lost to follow up, the rest died within 18 months of diagnosis. Adjuvant chemotherapy using gemcitabine or erlotinib have given marginal 1year survival of $24 \%{ }^{3}$. These drugs are expensive and not readily available in our environment, hence their efficacy is difficult to assess since our patients were unable to afford them.

\section{Conclusion:}

Pancreatic Cancer presents late in our environment and carries a poor prognosis. The incidence in our environment is low compared with the western world. Early presentation and diagnosis with curative Whipples operation offers the best prognosis.

\section{References}

[1] A BLowenfels, PMaisonneuve.Epidemiology and Prevention of Pancreatic Cancer.JpnJ. Clin. Oncol.34 (5),2004,238-244.

[2] EQ Archampong. Pancreas.In :EA Badoe, EQ Archampong, JT da-Rocha-Afodu,eds. Principles and Practice of Surgery -Including Pathology in the Tropics. $4^{\text {th }}$ Ed. Accra: Assemblies of God Literature Centre Ltd. 2009, 783-787

[3] CA Onyekwere, JC Ojukwu: The Epidemiologic profile of pancreatic cancer and management outcome in Lagos, Nigeria. Pak J Med Sci, 24(2),2008,340-343

[4] OG Ajao, AA Abioye. Pancreatic Carcinoma. J Nati Med Assoc 72(6),1980,580-596

[5] S Bhattacharya. The Pancreas. In NS Williams, CJK Bulstrode,P R O'Connell, eds. Bailey and Love's Short Practice of Surgery, $25^{\text {th }}$ Ed. London: Edward Arnold 2008,1148-1152 .

[6] J Smith III , C Baugh, T Reppun. Obstructive Jaundice Secondary to Pancreatic Adenocarcinoma in a seven year old male. J Pediatric Surg,20(2): $184-185$

[7] E K Wiredu, H B Armah. Cancer Mortality Patterns in Ghana: a 10-year review of autopsies and hospital mortality. BMC Public Health ,6,2006, 159

[8] M L Steer. Exocrine Pancreas.In M Courtney ,R Beauchamp ,B Evers ,K Mattox ,eds.Sabiston Textbook of Surgery, 17 ${ }^{\text {th }}$ Ed.Elsevier Saunders 2004,1667-74

[9] OI Alatise, OO Lawal, OT Ojo.Challenges of Pancreatic Cancer Management in a Resource Scarce Setting.East and Central African Journal of Surgery 15( 2),2010 52-58

[10] F G El Kamar, M L Grossbard ,P SKozuch.Metastatic Pancreatic Cancer: Emerging Strategies in Chemotherapy and Palliative Care. The Oncologist ,8(1),2003,18-34.

[11] E N Scott, G Garcea, H Doucas, W P Steward, A R Dennison, D P Berry. Surgical bypass vs. endoscopic stenting for pancreatic ductal adenocarcinoma. HPB (Oxford), 11(2),2009,118-124

[12] BA van Wagensveld, PP Coene, TM van Gulik, EA Rauws, H Obertop, DJ Gouma.Outcome of palliative biliary and gastric bypass surgery for pancreatic head carcinoma in 126 patients. Br J Surg,84(10),1997,1402-6

[13] A G Holbrook, J F Chester, and D C Britton.Surgical palliation for pancreatic cancer: will biliary bypass alone suffice? J R Soc Med 83(1),1990,12-14

[14] Manenti A. Unresectable Pancreatic Head Cancer: Double Palliative By-pass with a Single Roux-en.YJejunal Loop.WebmedCentral SURGICAL TECHNIQUE 20112(7),WMC002029 